\title{
Scientific Writing Studio: How publishers can help authors to write smarter and faster
}

\author{
Agnes Henri ${ }^{1, \star}$ and Charlotte Van Rooyen ${ }^{1, \star \star}$ \\ ${ }^{1}$ EDP Sciences, 17, av. du Hoggar - BP 112 - 91944 Les Ulis Cedex A, France
}

\begin{abstract}
Researchers are under pressure: publish or perish is one of their main challenges. Writing is hard and scientific writing is harder, and any help Publishers can provide to assist with the process is greatly appreciated, especially by younger authors. EDP Sciences and Astronomy \& Astrophysics (A\&A) have worked together to support their authors and developed a solution called Scientific Writing Studio (SWS) designed to make the article writing process easier. SWS is a tool that allows authors to collaboratively write their paper, then submit and then follow all stages of the publication process within a single unique interface. This paper explains the background to the development of the SWS, and describes the current benefits of the SWS as well as outlining future developments.
\end{abstract}

\section{Background}

A\&A has been publishing the best astronomical research for over 45 years and is the first choice for publication of important special issues including those from the missions of Planck, Herschel, Rosetta and Gaia (Fig. 1). This means there are often many contributors to the same paper (Fig. 2).

The Editors-in-Chief of A\&A conceived the idea of the SWS in 2013.

The Editors Dreamland (Fig. 3) was to create a collaborative writing tool where authors could work with their co-authors using journal templates and eventually be able to submit their articles directly to the Journal.

\section{Development}

The writing studio work group tested a number of online collaborative LaTeX writing tools, and considered ways of adapting these tools to the specific needs of A\&A. The key requirements at that time were: the usability of the interface, the ability to promote the A\&A brand, and the ease of integrating A\&A specific tools and services into the interface.

${ }^{\star}$ e-mail: agnes.henri@edpsciences.org ORCID: 0000-0001-7928-2060

$\star \star$ e-mail: charlotte.vanrooyen@edpsciences.org 


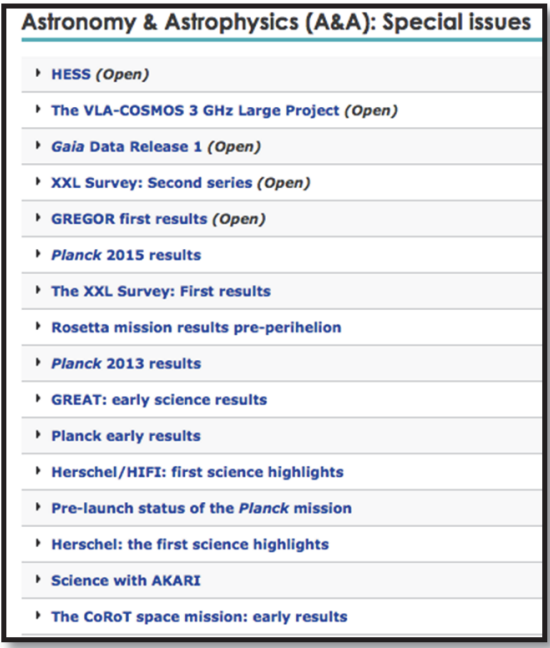

Figure 1. Some of the special issues published by Astronomy \& Astrophysics
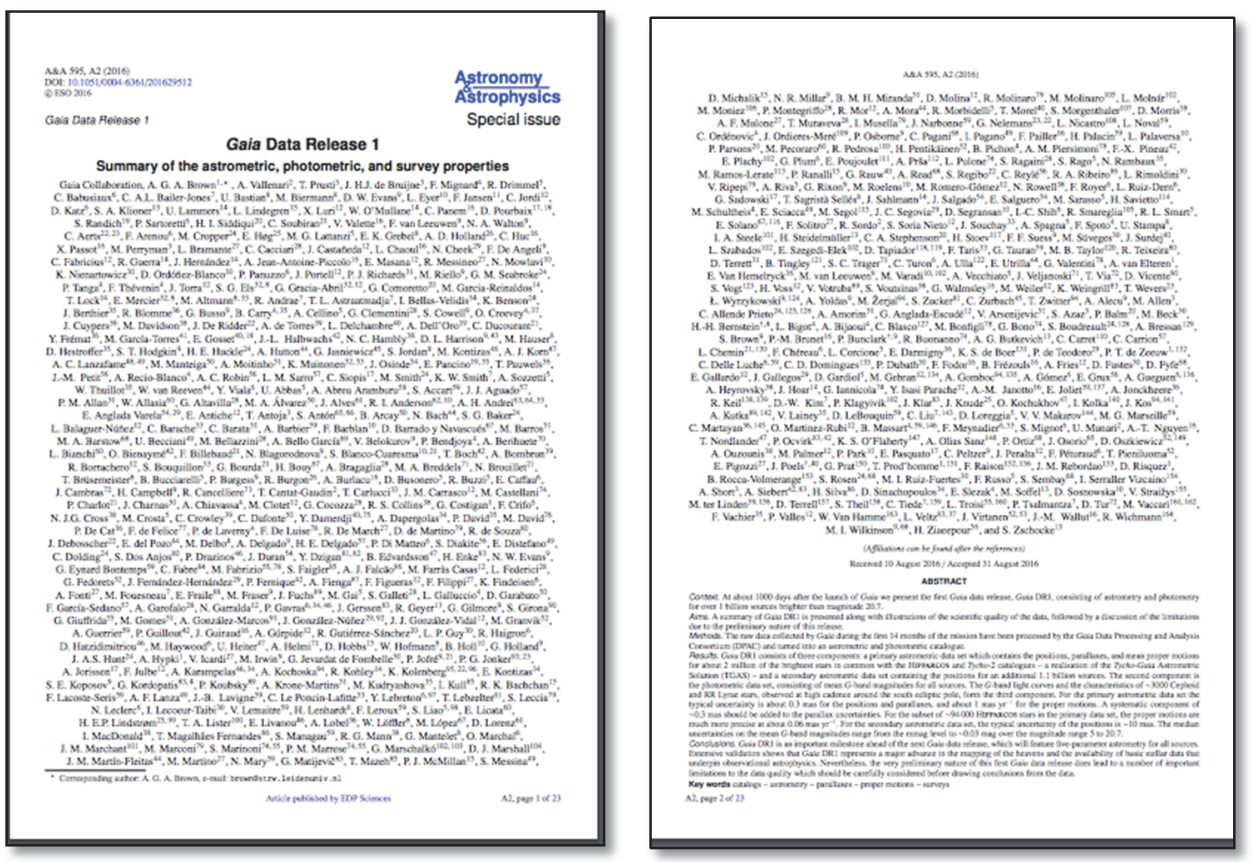

Figure 2. The many contributors to the Gaia Data Release 1. 


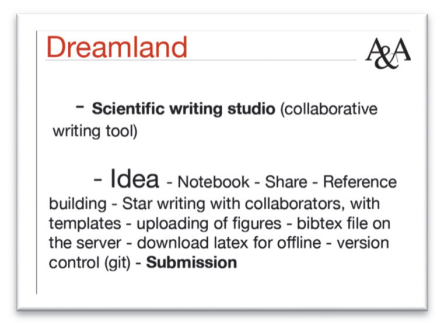

Figure 3. Writing Studio Dreamland.

The working group chose ShareLaTeX and a prototype was built using the ShareLaTeX opensource code supported under the open-source license that it uses. All changes and revisions were published to a Github public repository. In addition Dropbox sync functionality was provided under a license agreement to make this available to all users of the writing studio.

The tool underwent rigorous user-testing, gathering feedback from editors and beta testers to help design the best solutions and features to ensure the best user experience. The ambition was for the SWS to eventually provide a simple tool not just for authoring an article, but also to be able to submit to the journal. Additionally, it was required that the A\&A language editors should be able to use the tool to submit their corrections and edits.

\section{Making Scientific Writing Studio different}

This tool has been designed and developed specifically for authors of A\&A and those writing in the disciplines of astronomy and astrophysics. Specific features were developed for the SWS, for example EDP Sciences worked closely with CDS to include a tool in SWS that automatically detects and highlights stellar objects (see Fig. 4). Login using an ORCID iD was also integrated in order to authenticate login and ensure no ambiguity over author names, which is increasingly a problem in the scholarly research community.

\section{Launch}

The SWS was launched at the end of 2015 and now has well over 600 accounts with nearly 800 articles having been worked on. The Editors' ambition for a collaborative tool has been achieved as

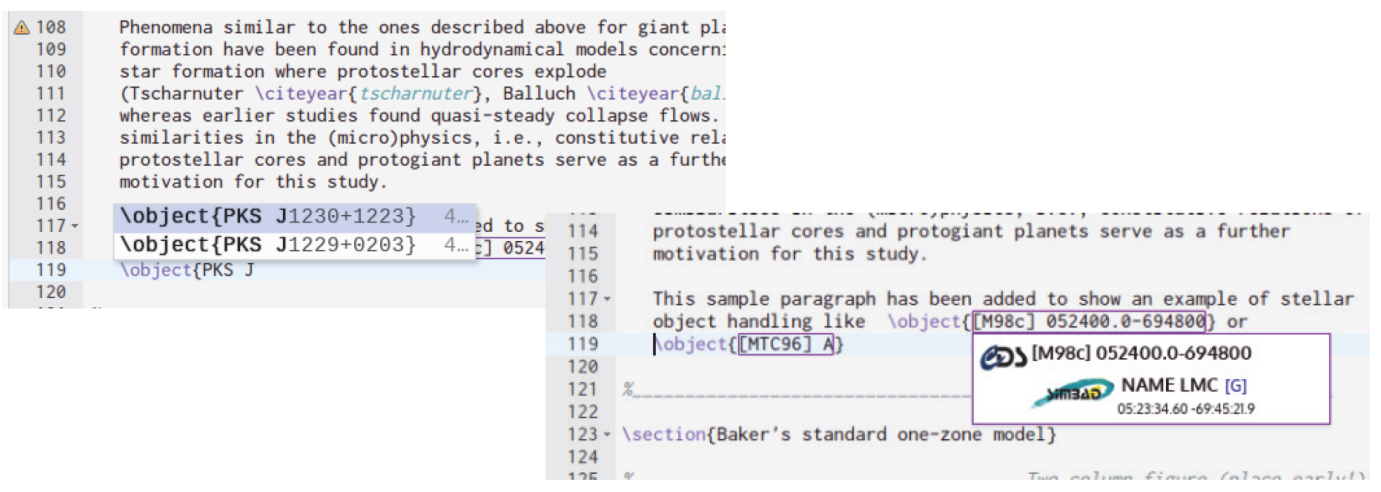

Figure 4. Links to stellar objects. 
Table 1. Some numbers

\begin{tabular}{lcc}
\hline & May 17 & August 16 \\
\hline Number of active accounts (connected at least once): & 511 & 323 \\
\hline Number of projects (total): & 738 & 355 \\
\hline Projects with at least one declared collaborator: & 170 & 70 \\
\hline Maximum number of collaborators on a project: & $16(1$ project) & 8 \\
\hline Number of files with more than 100 modifications: & 332 & 92 \\
\hline Chats around the projects: & 208 & 103 \\
\hline
\end{tabular}

up to 16 authors at a time have been working on a single paper, and nearly 200 with more than one collaborator! (Table 1).

\section{Overview of capabilities}

The SWS has been developed to allow authors to spend less time with LaTex and process, and more time on presenting and defending their science in collaboration with their co-authors. Today, the SWS offers authors the following benefits:

- A collaborative LaTeX solution: Author and co-authors can work on a unique version of the paper with A\&A macros already included.

- Easier links to stellar objects: Authors get auto-completion for stellar objects and additional information using tools developed with the CDS.

- Chat with co-authors: Authors are able to ask questions and chat with co-authors for direct communication.

- Dropbox integration: Articles can be edited offline and are automatically synchronized with the Dropbox online copy.

- ORCID integration: allows ease of login recognition to Writing Studio.

- Ease of Submission: automatic upload of article to submission system, with key fields pre-filled ready for checking.

All of these means SWS, provides a stronger bond between the journal and the author by making the writing of their articles easier, and that means they want to come back to the journal with the results of their research in the future (Fig. 5). 


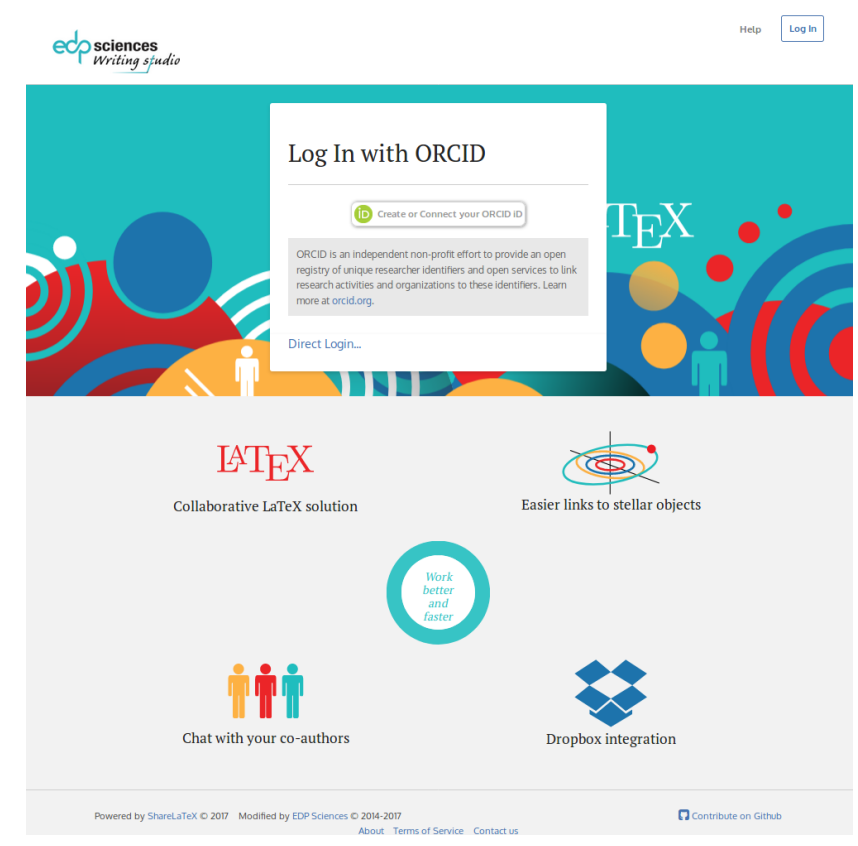

Figure 5. Login page for the Scientific Writing Studio.

\section{Future Developments}

Next steps are to:

- Integrate a citation helper to make building reference lists easy for the end user.

- The ability for an author to import from their ADS library papers they have bookmarked.

- Develop a comprehensive user guide.

\section{Conclusion}

By understanding the needs of researchers in a particular discipline, and working closely with the associated organisations in that field, as well as understanding the publishing process and services and tools to support publication, Publishers can assist authors to present their science in collaboration with their co-authors in an efficient way leaving more time for their research.

The SWS is bringing A\&A closer to their goal of a fully integrated and user-friendly workflow from writing through to submission and dissemination of an article (Fig.6). 

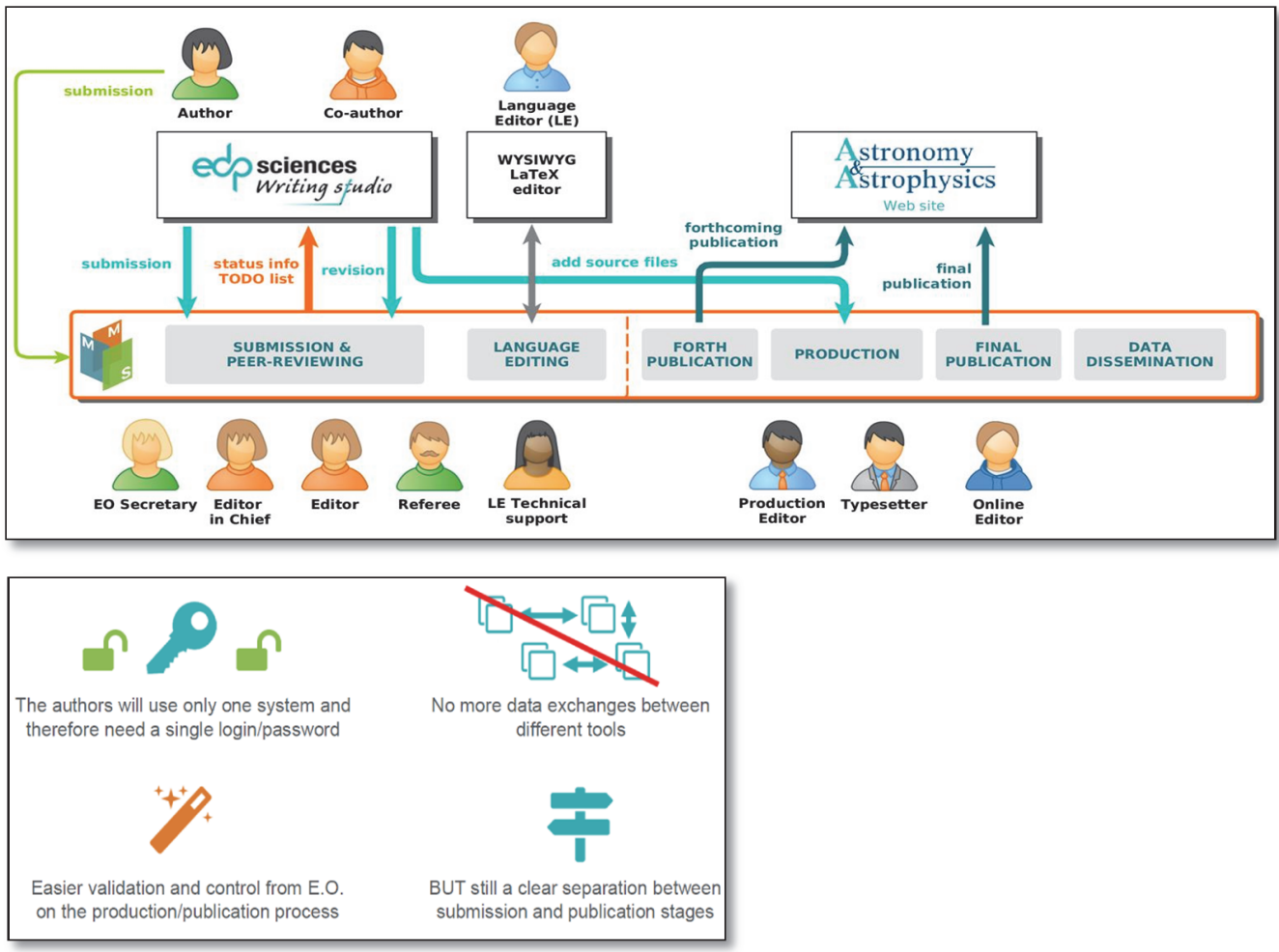

Figure 6. A\&A workflow. 